\title{
Diversidad, distribución y abundancia de especies en ensambles intermareales rocosos
}

\author{
Diversity, distribution and abundance of species in rocky intertidal assemblages
}

\author{
Patricio A. Camus ${ }^{1 *}$ \\ ${ }^{1}$ Departamento de Ecología Costera, Facultad de Ciencias, Universidad Católica de la Santísima Concepción, \\ Casilla 297, Concepción, Chile \\ pcamus@ucsc.cl
}

\begin{abstract}
Multiple sources of variation, including biogeographic processes, upwelling and El Niño, have differential effects on rocky intertidal communities over the north-central coast of Chile, and they could generate a high variability in diversity, particularly at a regional scale. In this context, species richness, dominance, evenness and composition were assessed in 17 sessile assemblages located between $18^{\circ}$ and $34^{\circ} \mathrm{S}$, along with the abundance and distribution of their 68 constituent species, for determining their patterns of variation at different spatial scales. Different analyses showed, among other results, that: (a) excepting species composition, the spatial variation of all diversity attributes at the assemblage level was explained almost entirely (83-100\%) by local factors; (b) the variation in species richness at the scale of the sampling unit $\left(0.25 \mathrm{~m}^{2}\right)$ explained more than $61 \%$ of the variance, reflecting an apparent pattern of saturation; (c) at a geographical scale, rarity in both distribution and abundance was the prevailing trait, and these two variables were positively correlated at all spatial scales; (d) common (core) species showed a low spatial variability in abundance and tended to be the same in all assemblages; and (e) taxonomic composition was the only attribute showing regional differences, related with a higher beta diversity and a higher level of rarity in northern Chile, which also appeared to be completely included within the Peruvian biogeographic Province. The results suggest that local and small-scale processes could be important determinants of diversity in these assemblages.
\end{abstract}

Key words: Species richness, abundance, rarity, spatial scale, Chile

\section{Introducción}

El estudio ecológico de las comunidades litorales en costas rocosas de Chile ha tenido un gran crecimiento en número, calidad y diversidad temática por casi cuatro décadas (Thiel et al. 2007), superando gradualmente algunos sesgos de conocimiento que limitaban la comprensión de sus patrones de diversidad. Estos se debían principalmente al predominio de estudios a escala

* Autor invitado
Resumen.- Múltiples fuentes de variación incluyendo procesos biogeográficos, la surgencia y El Niño, afectan diferencialmente a las comunidades intermareales rocosas en Chile centro-norte, y podrían generar una alta variabilidad en sus patrones de diversidad, particularmente a escala regional. En este contexto se evaluó la riqueza, dominancia, uniformidad y composición de 17 ensambles sésiles ubicados entre los $18^{\circ}$ y $34^{\circ} \mathrm{S}$, así como la abundancia y distribución de sus 68 especies constituyentes, para determinar sus patrones de variación en distintas escalas espaciales. Una serie de análisis mostró, entre otros resultados, que: (a) exceptuando la composición, la variación espacial de todos los atributos de diversidad a escala del ensamble fue explicada casi enteramente (83-100\%) por factores locales; (b) la variación en riqueza específica a escala de la unidad muestral $\left(0,25 \mathrm{~m}^{2}\right)$ explicó más del $61 \%$ de la varianza, y reflejó un patrón aparente de saturación; (c) a escala geográfica, la rareza en distribución y abundancia fueron los rasgos predominantes, y ambas variables se correlacionaron positivamente en todas las escalas espaciales; (d) las especies más comunes mostraron una baja variabilidad espacial en abundancia y tendieron a ser las mismas en todos los ensambles; y (e) la composición taxonómica fue el único atributo que reveló diferencias regionales, asociadas a una mayor diversidad beta y mayor nivel de rareza en Chile norte, la cual además estaría incluida completamente en la Provincia biogeográfica Peruana. Los resultados sugieren que los procesos locales y de pequeña escala podrían ser determinantes importantes de la diversidad en los ensambles sésiles.

Palabras clave: Riqueza de especies, abundancia, rareza, escala espacial, Chile

local y de corto plazo centrados en un bajo número de especies, procesos o factores, y a un esfuerzo de muestreo muy heterogéneo a través de la costa, enfocado en las zonas más cercanas a los centros de investigación (Santelices 1980, Fernández et al. 2000, Broitman et al. 2001, Camus 2001, Thiel et al. 2007). No obstante, hoy se dispone de un marco de conocimientos físicos y biológicos mucho más completo y en escalas de espacio y tiempo más amplias, posibilitando evaluaciones más generales. 
Una revisión multidisciplinaria del estado actual de conocimiento de la costa norte de Chile (Thiel et al. 2007), muestra que las principales determinantes de la diversidad comunitaria litoral actúan diferencialmente a través de la costa, y tienden a promover la variación entre comunidades. Entre estas fuentes de variación destacan dos procesos oceanográfico-climáticos que pueden afectar directamente a una o más comunidades, o que inciden en el transporte larval y dispersión de especies afectando su asentamiento o reclutamiento. Uno de ellos es la variación espacial en ocurrencia, frecuencia e intensidad de la surgencia costera, con efectos diferenciales entre especies, niveles tróficos, grupos funcionales o ensambles locales (e.g., Moreno et al. 1998, Vásquez et al. 1998, Camus \& Andrade 1999, Broitman et al. 2001, Nielsen \& Navarrete 2004, Lagos et al. 2005, Wieters 2005, Camus 2008, Lagos et al. 2008). Otro es El Niño-Oscilación del Sur, que afecta diferencialmente la riqueza, abundancia y composición de las comunidades del intermareal rocoso, pero a su vez puede modificar la frecuencia e intensidad de la surgencia (e.g., Camus \& Ojeda 1992, Castilla \& Camus 1992, Camus 1994, Moreno et al. 1998, Navarrete et al. 2002, Takesue et al. 2004, Thiel et al. 2007, Camus 2008, Camus et al. 2008). Datos recientes de la composición de cuatro ensambles intermareales del norte de Chile entre $21^{\circ}$ y $30^{\circ} \mathrm{S}$, muestran que su nivel de recambio de especies es muy alto en espacio (46-57\% entre pares de ensambles, media $\sim 50 \%$; Camus, datos no publicados) y tiempo (promedio estacional $40-50 \%$ en cada ensamble; Camus 2008), lo cual podría ser una característica de las comunidades en esta región. Por otra parte, la interacción entre estos y otros factores resultaría en patrones distintivos de diversidad a mesoescala o a escala regional, que podrían ser más evidentes al comparar áreas con diferentes regímenes físico-ecológicos como las costas central y norte de Chile (Thiel et al. 2007). No obstante, a este nivel de comparación se agrega otro factor derivado de considerar una gran escala espacial, que es la mayor probabilidad de que los patrones comunitarios observados incluyan efectos biogeográficos (e.g., Menge \& Olson 1990, Ricklefs \& Schluter 1993), lo cual tiene especial interés en Chile centro-norte. En esta área, la biota templada-cálida de la Provincia Peruana disminuye hacia el sur a la vez que aumentan los componentes transicionales y templado-fríos, y mientras la mayoría de los taxa termina su distribución en $30^{\circ}$ o $33^{\circ} \mathrm{S}$ otros muestran puntos finales más variables en tiempo y espacio, aunque estos límites no tienen relación clara con sus rangos de tolerancia o patrones de endemismo (Camus 2001, Thiel et al. 2007, Ibáñez et al. 2008).

Todo lo anterior sugiere que la costa norte de Chile es un área altamente dinámica y heterogénea en términos ecológicos y biogeográficos, donde los forzamientos físicos jugarían un rol importante como determinantes de la composición comunitaria. Por ello es posible que las comunidades intermareales de las costas norte y central de Chile muestren patrones contrastantes en uno o más componentes básicos de su diversidad, o en sus patrones de rareza y diversidad beta. No obstante, los análisis de este tipo, involucrando escalas amplias y ensambles completos, son todavía muy escasos, particularmente para la zona norte (Thiel et al. 2007). En tal contexto, este trabajo presenta une caracterización de la diversidad y patrones de variación de 17 ensambles sésiles de costas intermareales rocosas en Chile centro-norte $\left(18-34^{\circ} \mathrm{S}\right)$, mediante un conjunto de descriptores relevantes evaluados a distintas escalas espaciales. Siguiendo un criterio geográfico, los ensambles fueron clasificados $a$ priori en dos grupos definidos como región norte $\mathrm{y}$ central, con el objetivo global de establecer el ámbito y estructura jerárquica de su variación en diversidad en función de la riqueza, distribución, abundancia y composición de sus especies constituyentes. La información aquí presentada fue recolectada hace cerca de 15 años, por lo cual refleja el estado del sistema de estudio en ese momento, y podría tener utilidad potencial como referente de comparación con patrones actuales.

\section{Material y métodos}

\section{Muestreo}

La unidad de estudio fue el ensamble de especies sésiles encontrado sobre la franja de Lessonia nigrescens Bory que marca la transición hacia el submareal (Castilla 1981, Santelices et al. 1981, Santelices \& Ojeda 1984), y que co-ocurren en esta zona mostrando diferencias en abundancia relativa en el gradiente vertical más que una zonación marcada (Camus et al. 1994, Camus 1998). Se incluyó a todas las especies ocupando substrato rocoso a nivel macroscópico y mensurable, principalmente macroalgas, filtradores, suspensívoros y detritívoros, que son usuarios obligados de espacio y conforman una matriz biológica que es la base estructural del sistema intermareal. En este sentido se les asignó el término ensamble en lugar de otros afines como taxoceno (e.g., Pielou 1976), grupo funcional (e.g., Littler \& Littler 1980), o gremio (e.g., Jaksic 1981).

Se seleccionaron 17 ensambles entre los $18^{\circ}$ y $34^{\circ} \mathrm{S}$ en costas rocosas expuestas al oleaje, nueve de ellos asignados a la región norte y ocho a la región central, dominados principalmente por cirripedios, algas clorófitas frondosas o mitílidos. Por problemas de accesibilidad y presencia de sectores de playas de arena del orden de $10^{1}-10^{2} \mathrm{~km}$, no se pudo lograr un espaciamiento regular 
Tabla 1

Localidades de estudio, ubicación en el contexto geográfico de muestreo, y abreviatura usada en Figuras. Chucumata corresponde a la franja costera del Aeropuerto Diego Aracena de Iquique, ECIM a la Estación Costera de Investigaciones Marinas de la P. Universidad Católica de Chile en Las Cruces, y Mejillones al sector de Caleta Errázuriz en la costa oeste de la Península de Mejillones. Zona: ciudad más cercana a cada localidad muestral, indicada como referencia. $N$ : zona norte de Chile; C: zona central de Chile

Study localities, their location in the geographical context of sampling, and their abbreviation as used in Figures. Chucumata corresponds to the coastal stretch of the Diego Aracena Airport at Iquique, ECIM to the Estación Costera de Investigaciones Marinas of the P. Universidad Católica de Chile at Las Cruces, and Mejillones to the site of Caleta Errázuriz at the west coast of the Mejillones Peninsula. Zona: nearest city to each

sampling locality, indicated as a reference. $\mathrm{N}$ : northern zone of Chile; $\mathrm{C}$ : central zone of Chile

\begin{tabular}{ccccc}
\hline Región & Zona & Localidad & Latitud & Abreviatura \\
\hline $\mathrm{N}$ & Arica & Arenillas Negras & $18^{\circ} 29^{\prime} \mathrm{S}$ & ANEG \\
$\mathrm{N}$ & Arica & Ind. Pesqueras & $18^{\circ} 30^{\prime} \mathrm{S}$ & IPES \\
$\mathrm{N}$ & Iquique & Los Verdes & $20^{\circ} 18^{\prime} \mathrm{S}$ & LOSV \\
$\mathrm{N}$ & Iquique & Chucumata & $20^{\circ} 32^{\prime} \mathrm{S}$ & AERO \\
$\mathrm{N}$ & Iquique & Aguadita & $20^{\circ} 36^{\prime} \mathrm{S}$ & AGUA \\
$\mathrm{N}$ & Iquique & Yape & $20^{\circ} 40^{\prime} \mathrm{S}$ & YAPE \\
$\mathrm{N}$ & Iquique & Chanavayita & $20^{\circ} 42^{\prime} \mathrm{S}$ & CHAN \\
$\mathrm{N}$ & Iquique & Patache & $20^{\circ} 48^{\prime} \mathrm{S}$ & PATA \\
$\mathrm{N}$ & Antofagasta & Mejillones & $23^{\circ} 24^{\prime} \mathrm{S}$ & MEJI \\
$\mathrm{C}$ & Pichidangui & Los Molles & $32^{\circ} 13^{\prime} \mathrm{S}$ & LOSM \\
$\mathrm{C}$ & Valparaíso & Cón Cón & $32^{\circ} 54^{\prime} \mathrm{S}$ & CONC \\
$\mathrm{C}$ & Valparaíso & Montemar & $32^{\circ} 59^{\prime} \mathrm{S}$ & RENA \\
$\mathrm{C}$ & San Antonio & El Quisco & $33^{\circ} 23^{\prime} \mathrm{S}$ & ELQU \\
$\mathrm{C}$ & San Antonio & El Tabo & $33^{\circ} 27^{\prime} \mathrm{S}$ & ELTA \\
$\mathrm{C}$ & San Antonio & ECIM & $33^{\circ} 30^{\prime} \mathrm{S}$ & ECIM \\
$\mathrm{C}$ & San Antonio & Las Cruces & $33^{\circ} 30^{\prime} \mathrm{S}$ & LASC \\
$\mathrm{C}$ & San Antonio & Pelancura & $32^{\circ} 32^{\prime} \mathrm{S}$ & PELA \\
\hline
\end{tabular}

entre los sitios, por lo cual se decidió no aplicar estadística espacial para no forzar los supuestos usuales en estos análisis, y se privilegió una adecuada representación de las regiones central y norte. La Tabla 1 indica el nombre y ubicación geográfica de las localidades estudiadas, junto a las abreviaturas por las que serán referidas en algunas figuras.

Los muestreos se realizaron en un período cercano a dos años entre mediados de 1991 y mediados de 1993. En cada uno de los dos años se evaluó el conjunto completo de ensambles usando el mismo diseño muestral, pero invirtiendo la secuencia temporal de muestreo entre años a fin de recoger las eventuales variaciones estacionales. Para evitar problemas por la agregación o desagregación de unidades muestrales (Hurlbert 1984), en cada localidad se efectuó un muestreo sistemático midiendo dos series paralelas de cuadrantes de $0,25 \mathrm{~m}^{2}$ siguiendo la línea de costa, ubicadas entre la franja de $L$. nigrescens y el límite intermareal superior. Ambas series estuvieron espaciadas ca. $1 \mathrm{~m}$ en distancia vertical, y el espaciamiento entre cuadrantes de cada serie fue de ca. 1,5 m. Muestreos preliminares en Las Cruces y Chanavayita (Tabla 1) mostraron que el punto de inflexión en una curva de acumulación de especies variando el área del cuadrante $\left(0,01 \mathrm{a}^{1} \mathrm{~m}^{2}\right)$ fue de $0,16 \mathrm{~m}^{2}$, por lo cual se eligió una unidad de $0,25 \mathrm{~m}^{2}$ para asegurar la representatividad muestral. El tamaño mínimo de muestra estimado por la minimización del error estándar del promedio de riqueza de especies (Bros \& Cowell 1987) fue $n=25$, pero se usó siempre un $n>35$ para evitar correlaciones entre el atributo medido y el tamaño de la muestra, con una media de 46,2 \pm 7,3 (d.e.) cuadrantes por localidad. Una evaluación previa de los resultados mostró que el tamaño de muestra no se correlacionó con el número promedio $\left(\mathrm{r}_{\text {Spearman }}=0,240, P=0,34, \mathrm{n}=17\right) \mathrm{o}$ total $\left(\mathrm{r}_{\text {Spearman }}=0,437, P=0,08, \mathrm{n}=17\right)$ de especies en los ensambles, sugiriendo que no habrían sesgos relevantes. En cada cuadrante se registró el número e identidad de las especies presentes, y el porcentaje de cobertura de las especies sésiles (100 puntos de intersección; Castilla 1988) en forma visual en vez de fotográfica, lo cual permite mayor precisión y menor sesgo con tamaños de muestra altos (Dethier et al. 1993). La cobertura consideró el sustrato primario y secundario ya que la mayoría de las especies sésiles los usa indistintamente, y el último es un factor relevante para disminuir las interacciones potenciales por espacio (e.g., Lohse 1993).

\section{Análisis de diversidad}

El grado de asociación entre variables se analizó mediante correlación paramétrica (Pearson, $r_{p}$ ) o no paramétrica (Spearman, $\mathrm{r}_{\mathrm{s}}$ ), dependiendo de la existencia de normalidad y/o linealidad en cada caso. La importancia cuantitativa del ensamble sésil se evaluó en relación a la riqueza total de especies registrada en cada localidad. Las asociaciones entre el número de especies sésiles, su proporción respecto al total de especies, y el número total de especies, se analizaron considerando el conjunto de comunidades. La variación espacial de la proporción de especies sésiles respecto al total (datos transformados por arcoseno) se evaluó a escala regional (central $v s$. norte) y local (ensambles) mediante análisis de varianza (ANDEVA) jerárquico, estimando el componente de varianza de cada nivel (contribución relativa a la varianza total), y docimando su efecto respecto al nivel inferior mediante la razón de F (Sokal \& Rohlf 1995; en los ANDEVA jerárquicos debió usarse un número balanceado de sitios por región, por lo cual en el norte se excluyó 
aquel con menor tamaño muestral: Chucumata). Además, se evaluó indirectamente la posibilidad de que la riqueza del ensamble sésil alcanzara un nivel de saturación, para lo cual se examinó visualmente la variación de la proporción de especies sésiles en función de la riqueza total a escala de la unidad muestral. Además, se analizó un análogo a menor escala de la relación entre la riqueza local y la riqueza regional de especies (véase Cornell 1993), estimando la riqueza promedio de las unidades muestrales de cada ensamble ('local') y su relación con la riqueza total en el ensamble respectivo ('regional'). A fin de predecir el grado máximo de saturación esperable, a esta relación se ajustó una ecuación de tasa de primer orden $\left(\mathrm{y}=\mathrm{c} *\left(1-\mathrm{e}^{(-\mathrm{kt})}\right)+\mathrm{d}\right)$ usada para cinética enzimática con parámetros sin significado ecológico, con el único propósito de estimar el límite asintótico de la riqueza 'local' en este contexto.

Para comparar las localidades y regiones entre sí se estimó la riqueza de especies, y la dominancia y uniformidad (distribución relativa de cobertura) de las especies en cada ensamble, medidas por índices que corrigen sesgos por diferencias en número de muestras o número de especies entre muestras (Ludwig \& Reynolds 1988, Magurran 1988). La dominancia se evaluó usando N2 (recíproco del estimador insesgado de $\lambda$ de Simpson; Hill 1973) que estima el número de especies muy abundantes en una comunidad (Ludwig \& Reynolds 1988), y la uniformidad usando E5 (corregido de una medida Hill y basada en N2 y el exponencial de Shannon; Ludwig \& Reynolds 1988), que tiende a cero a medida que una especie aumenta su importancia numérica (i.e. al disminuir la uniformidad). Estos tres aspectos fueron evaluados estadísticamente mediante ANDEVA jerárquicos, determinando los componentes de varianza de distintas escalas espaciales: región, ensamble y unidad muestral para la riqueza de especies, y región y ensamble para la dominancia y uniformidad estimadas a nivel del ensamble (i.e. un valor por ensamble).

En otro contexto, se evaluó la relación entre la distribución y abundancia de las especies en tres escalas distintas por análisis de correlación. La distribución de cada especie se definió para cada escala como: (a) local: proporción del total de unidades muestrales en que estuvo presente; (b) regional: proporción del total de localidades en que se encontró en cada región; y (c) geográfica: ocurrencia en el total de localidades muestreadas. La abundancia de cada especie se expresó como su promedio de cobertura en el total de unidades muestrales a escala local, el total de localidades a escala regional, y el total de localidades a escala geográfica. Adicionalmente, a escala geográfica y para el conjunto de especies sésiles estudiadas, se efectuó una caracterización de tres aspectos relacionados: (a) distribución de frecuencia de la distribución de especies (i.e. número de especies ocurriendo en distintos intervalos de clase de distribución) para determinar las especies más comunes y raras, clasificándolas como núcleo o satélite (Hanski 1982) si ocupan respectivamente al menos $90 \%$ y a lo más $10 \%$ de las localidades; (b) variabilidad espacial de la abundancia de las especies, estimada por su coeficiente de variación en cobertura entre ensambles (incluyendo valores cero según el criterio de Wolda \& Marek 1994), para evaluar su asociación con la distribución y la abundancia local promedio; y (c) curva dominanciadiversidad de Chile centro-norte, i.e. cobertura promedio (escala logarítmica) versus ranking de abundancia de cada especie en orden decreciente, para compararla con la forma predicha por modelos clásicos de distribución de abundancia, en particular el lognormal (Preston 1948) debido al número de especies y ensambles involucrados (véase Magurran 1988).

La variación en composición taxonómica de los ensambles se evaluó mediante análisis de afinidad (medida estandarizada de suma de rangos derivada del índice de Jaccard), que analiza el arreglo relativo de subunidades ecológicas dentro de una unidad mayor en función de la presencia de especies raras y comunes, diferencias en riqueza entre sitios, y la interacción entre ambos aspectos (Istock \& Scheiner 1987, Scheiner 1992). El cálculo de la afinidad media entre todos los ensambles permite derivar un estimador de la complejidad composicional del paisaje (diversidad de mosaico), y definir aquellos ensambles sobre o bajo 1 d.e. de la media respectivamente como modales (cuya composición es la más representativa del total de sitios) y extremos (con más especies no representadas en los demás sitios). Los valores observados se compararon contra tres modelos nulos generados por remuestreo aleatorio de la matriz original de especies (filas) por sitios (columnas), simulando tres efectos: (a) dispersión aleatoria de las especies, restringiendo (fijando) el total de la matriz; (b) 'capacidad de carga' de especies en cada localidad, fijando el total de las columnas; y (c) distribución inherente de cada especie basada en su ocurrencia actual, fijando el total de las filas. Los valores esperados y observados de similitud media y diversidad de mosaico se compararon mediante una prueba $t$ de Student.

\section{Resultados}

Debido a la extensión de los resultados, esta sección presenta una síntesis de los aspectos principales, privilegiando su descripción en el texto más que en tablas o figuras, en las cuales se mostrarán principalmente aquellos menos frecuentes o menos analizados en la literatura. 


\section{Importancia numérica de las especies sésiles}

A escala geográfica se registró un total de 68 especies sésiles, 56 y 51 en la región norte y central respectivamente, que representaron en promedio el 65,0 $\pm 5,8 \%$ (d.e.) de las especies de cada ensamble, reflejando su importancia numérica y estructural. El número total de especies (sésiles + móviles) por ensamble fue en promedio de 41,5 $\pm 6,9$ (d.e.), con un coeficiente de variación de $16,5 \%$, y el número de especies sésiles de $26,9 \pm 4,8$ (d.e.), con un coeficiente de variación de 17,7\%. La Fig. 1 muestra el número y proporción de especies sésiles por ensamble (ordenados de norte a sur), y su aparente falta de tendencia en función de la latitud. Esta proporción mostró un bajo coeficiente de variación entre ensambles $(8,9 \%)$, sin correlación con el número total de especies $\left(\mathrm{r}_{\mathrm{s}}=-0,119 ; P=0,634 ; \mathrm{n}=17\right)$ ya que el número de especies sésiles a su vez varió positivamente con el número total $\left(\mathrm{r}_{\mathrm{p}}=0,904 ; P<0,0001 ; \mathrm{n}=17\right)$. Un ANDEVA jerárquico (Tabla 2) mostró que la proporción de especies sésiles no fue significativamente distinta entre la región norte y central, mientras que la variación entre ensambles explicó más del $80 \%$ de la varianza dentro de cada región (i.e. la variación observada se asocia principalmente a factores locales).

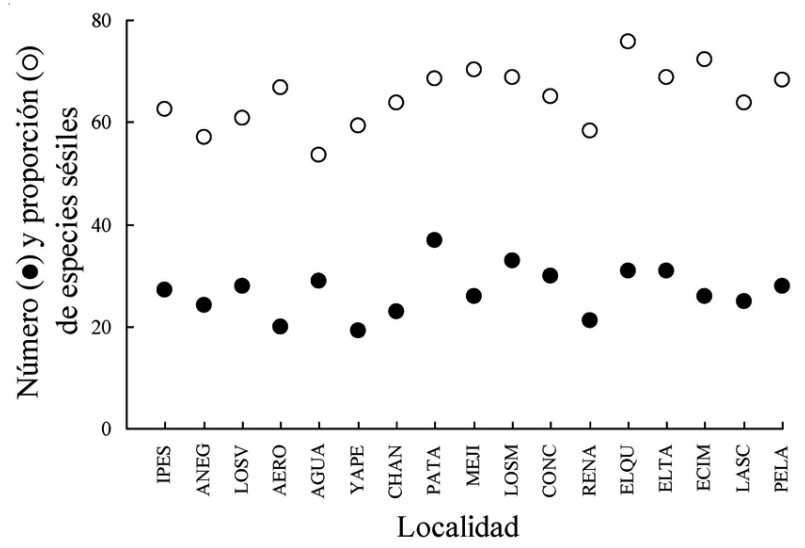

Figura 1

Número de especies sésiles y su proporción (\%) respecto a la riqueza total de especies en los ensambles del área de estudio, ordenados de norte a sur (véase abreviaciones en Tabla 1)

Number of sessile species and its proportion (\%) with respect to the total species richness in the assemblages of the study area, ordered from north to south (see abbreviations in Table 1)

\section{Tabla 2}

Análisis de varianza jerárquico a escala regional (región norte vs. central) para cuatro variables estimadas a nivel del ensamble, describiendo la diversidad de especies sésiles en el área de Chile centro-norte: cuociente entre el número de especies sésiles y el número total de especies (Proporción), número de especies sésiles (Riqueza), índice N2 de Hill (Dominancia), y razón modificada de Hill E5 (Uniformidad).

Primera columna: variables dependientes (en mayúsculas)

y fuentes de variación; gl: grados de libertad; $C M$ : cuadrado medio; $\mathrm{CdV}$ : componente de varianza

Hierarchical analyses of variance at regional scale (northern $v s$. central region) of four variables estimated at the assemblage level, describing the diversity of sessile species in the centralnorthern Chilean area: quotient between the number of sessile species and the total number of species (Proporción: proportion), number of sessile species (Riqueza: richness), Hill's N2 index (Dominancia: dominance), and Hill's modified ratio E5 (Uniformidad: evenness). First column: dependent variables (upper case) and sources of variation (region and assemblage); gl: degrees of freedom; CM: mean square; $\mathrm{CdV}$ : variance component

\begin{tabular}{|c|c|c|c|c|c|}
\hline & gl & $\mathrm{CM}$ & $\mathrm{F}$ & $P$ & $\mathrm{CdV}(\%)$ \\
\hline $\begin{array}{l}\text { PROPORCIÓN } \\
\text { Región } \\
\text { Región (Ensamble) }\end{array}$ & $\begin{array}{r}1 \\
14\end{array}$ & $\begin{array}{l}0,0072 \\
0,0027\end{array}$ & 2,66 & 0,15 & $\begin{array}{l}17,2 \\
82,8\end{array}$ \\
\hline $\begin{array}{l}\text { RIQUEZA } \\
\text { Región } \\
\text { Región (Ensamble) }\end{array}$ & $\begin{array}{r}1 \\
14\end{array}$ & $\begin{array}{r}9,00 \\
21,63\end{array}$ & 0,146 & 0,70 & $\begin{array}{r}0 \\
100\end{array}$ \\
\hline $\begin{array}{l}\text { DOMINANCIA } \\
\text { Región } \\
\text { Región (Ensamble) }\end{array}$ & $\begin{array}{r}1 \\
14\end{array}$ & $\begin{array}{l}0,0065 \\
0,0120\end{array}$ & 0,858 & 0,40 & $\begin{array}{r}0 \\
100\end{array}$ \\
\hline $\begin{array}{l}\text { UNIFORMIDAD } \\
\text { Región } \\
\text { Región (Ensamble) }\end{array}$ & $\begin{array}{r}1 \\
14\end{array}$ & $\begin{array}{l}4,78 \\
5,57\end{array}$ & 0,542 & 0,45 & $\begin{array}{r}0 \\
100\end{array}$ \\
\hline
\end{tabular}

A escala de la unidad muestral, y considerando todos los cuadrantes analizados en los 17 ensambles, se observó que cuando el número total de especies es bajo, la proporción de especies sésiles es muy variable y puede alcanzar altos valores, incluso $100 \%$ en muchos casos (Fig. 2). Sin embargo, a medida que la riqueza total aumenta la proporción de especies sésiles tiende a converger a un rango acotado de valores, que en la Fig. 2 corresponden a $65-70 \%$, similares al promedio registrado a escala del ensamble. Esto indica que cuando la riqueza total se acerca a sus valores máximos, el número de especies sésiles continúa aumentando pero a una tasa decreciente, sugiriendo una saturación estructural a pequeña escala. 


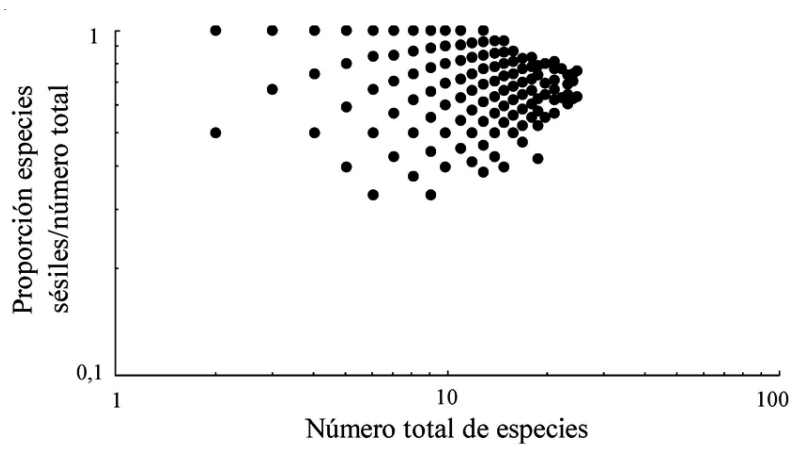

Figura 2

Relación entre la proporción de especies sésiles y el número total de especies registrados a escala de la unidad muestral, considerando todas las unidades medidas en los ensambles de estudio (en escala logarítmica para facilitar la visualización)

Relationship between the proportion of sessile species and the total number of species recorded at the scale of the sampling unit, considering all units measured in the study assemblages (in logarithmic scale for improving visualization)

\section{Variación espacial de la riqueza y abundancia de especies sésiles}

La riqueza de especies sésiles presentó una alta variación a través del área de estudio (Fig. 1), y tanto su máximo como su mínimo estuvieron en localidades de la región norte (37 en Patache, 19 en Yape) la cual aparece más heterogénea que la región central. Sin embargo, el ANDEVA jerárquico (Tabla 2) mostró que la riqueza de especies sésiles no fue significativamente diferente entre regiones, y además que el 100\% de la variación observada se debió a diferencias entre localidades, resaltando la relevancia de los factores locales sobre los regionales. Por otra parte, la dominancia y uniformidad de los ensambles siguió un patrón similar al de la riqueza específica. Ambos atributos, y en especial la dominancia, fueron muy variables en toda el área de estudio y entre localidades cercanas (Fig. 3), y se asociaron positivamente $\left(\mathrm{r}_{\mathrm{p}}=0,732 ; P=0,0008 ; \mathrm{n}=17\right)$ ya que la uniformidad tiende a aumentar si hay más especies dominantes. En ambos casos los ANDEVA jerárquicos (Tabla 2) no detectaron diferencias significativas entre regiones, $\mathrm{y}$ al igual que en el caso de la riqueza, el componente de varianza de la escala local alcanzó el 100\%.

Los resultados anteriores sugieren que la riqueza y abundancia relativa de los ensambles de Chile centronorte tendrían un carácter más bien idiosincrásico, dependiente de los factores locales, aunque esto se relativiza en parte al cambiar el nivel de resolución

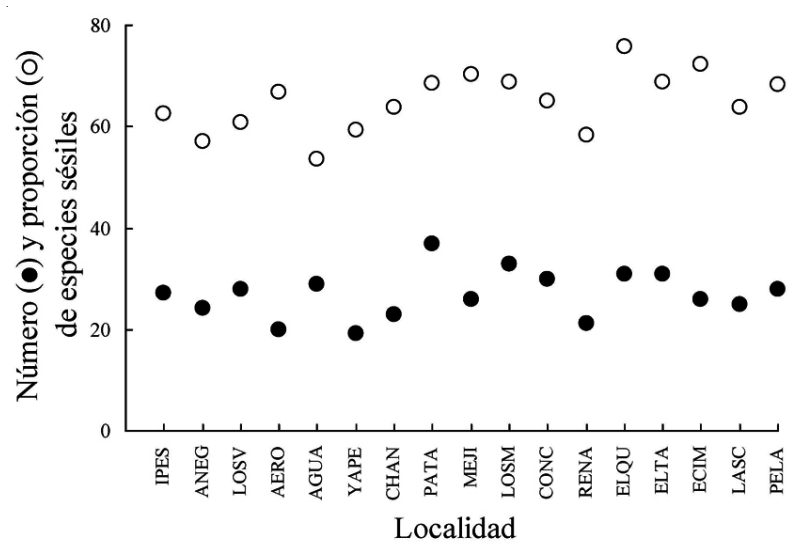

Figura 3

\author{
Dominancia (índice N2 de Hill) y uniformidad (razón \\ modificada de Hill E5) de los ensambles sésiles en \\ el área de estudio, ordenados de norte a sur \\ (véase abreviaciones en Tabla 1)
}

Dominance (Hill's N2 index) and evenness (Hill's modified ratio E5) of the sessile assemblages in the study area, ordered from north to south (see abbreviations in Table 1)

espacial. El ANDEVA jerárquico para la riqueza específica registrada a escala de la unidad muestral (Tabla 3) no mostró diferencias entre las regiones norte y central, pro sí un efecto significativo de la variación entre localidades dentro de cada región. Sin embargo, la escala regional explicó sólo 5\% de la varianza total, contra $\sim 33,5 \%$ de la escala local y $\sim 61,5 \%$ de la escala de la unidad muestral, indicando que los factores de pequeña escala serían tanto o más importantes que los locales para explicar la diversidad de los ensambles sésiles.

Por otra parte, la relación entre riqueza promedio de la unidad muestral y riqueza total del ensamble fue positiva y significativa $\left(\mathrm{r}_{\mathrm{p}}=0,865 ; P=0,00001 ; \mathrm{n}=17\right)$, sugiriendo un efecto de muestreo proporcional (Fig. 4). No obstante se ajustó bien $\left(\mathrm{R}^{2}=89 \% ; P<0,0001\right)$ a una curva no lineal con tasa decreciente, que predice una riqueza intra ensamble de 11,04 especies para un ensamble de 40 especies, similar a lo observado en el ensamble más diverso (Patache) con 10,93 y 37 especies respectivamente, y se estabiliza en 12,8 para un ensamble de 95 o más especies (i.e. el número promedio de especies sésiles por unidad muestral tendría un máximo de 13). En este contexto, la situación en que la proporción de especies sésiles se estabiliza en 65-70\% sería el umbral en que la unidad muestral aproxima su valor límite de riqueza, sugiriendo que al menos los ensambles más diversos podrían haber alcanzado una saturación a pequeña escala. 


\section{Tabla 3}

Análisis de varianza jerárquico de la riqueza de especies sésiles en el área Chile centro-norte estimada a nivel de la unidad muestral. Escalas espaciales: Región (norte vs. central), Ensamble (local), Unidad muestral. Abreviaciones igual que en Tabla 2

Hierarchical analyses of variance of the richness of sessile species in the central-northern Chilean area estimated at the sampling unit level. Spatial scales: Región (region: northern vs. central), Ensamble (assemblage: local),

Unidad muestral (sampling unit). For abbreviations see Table 2

\begin{tabular}{lrrccr}
\hline Escala espacial & gl & \multicolumn{1}{c}{$\mathrm{CM}$} & \multicolumn{1}{c}{$\mathrm{F}$} & $P$ & $\mathrm{CdV}$ \\
\hline Región & 1 & 280,56 & 2,13 & 0,20 & 4,99 \\
Región (Ensamble) & 14 & 131,56 & 20,62 & $<0,001$ & 33,52 \\
Región (Ensamble (Unidad muestral) & 560 & 6,38 & & & 61,49 \\
\hline
\end{tabular}

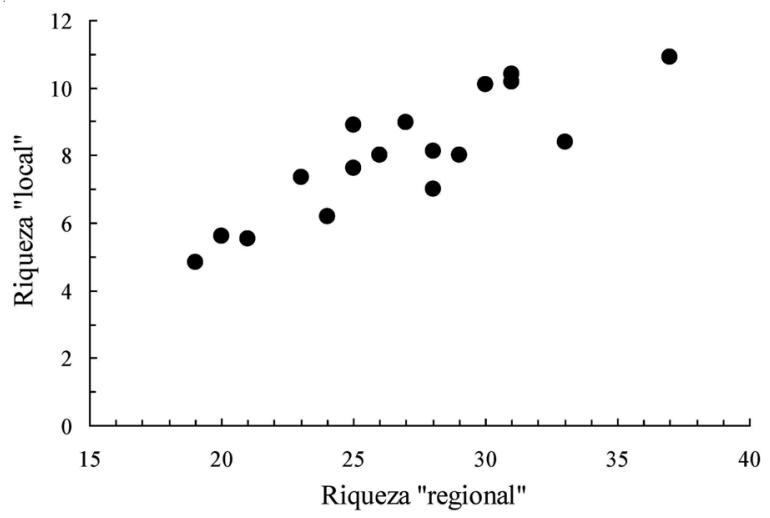

Figura 4

Relación entre el número promedio de especies sésiles en las unidades muestrales dentro de cada ensamble (riqueza 'local') y el número total de especies sésiles del ensamble respectivo (riqueza 'regional')

Relationship between the mean number of sessile species within each assemblage ('local' richness) and the total number of sessile species of the respective assemblage

('regional' richness)

\section{Distribución, abundancia y rareza}

La forma de la curva de dominancia-diversidad de las especies sésiles a escala geográfica (Fig. 5) fue más plana y alargada que una lognormal típica, y distinta a las del tipo serie logarítmica, serie geométrica o barra quebrada. La diferencia se debe al número relativamente bajo de especies con dominancia intermedia, y a un alto número de especies raras que estarían bien representadas en el muestreo, reduciendo el sesgo asociado a la 'línea de velo'. La frecuencia de especies sésiles respecto a la proporción de localidades ocupadas a escala geográfica (Fig. 6), muestra que un alto número de especies (35;

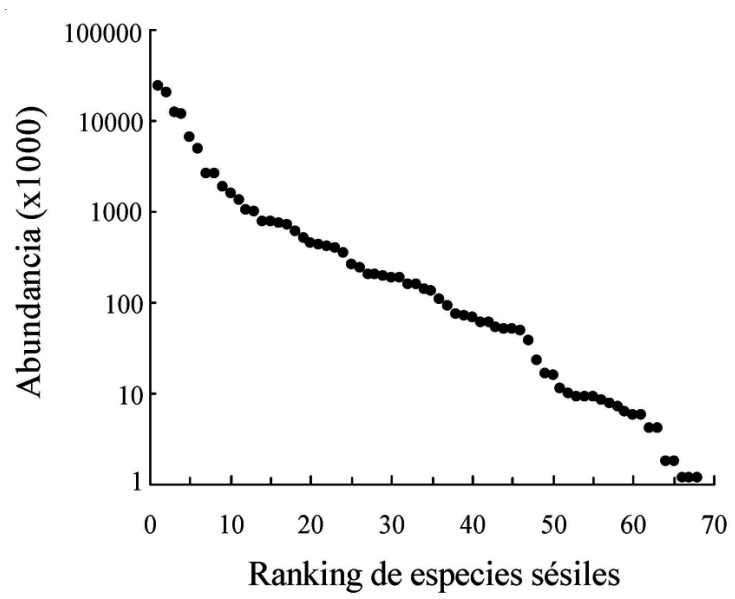

Figura 5

Curva de dominancia-diversidad para las 68 especies sésiles registradas en Chile centro-norte: abundancia (cobertura) geográfica promedio versus ranking de abundancia

Dominance-diversity curve for the 68 sessile species recorded in northern-central Chile: mean geographical abundance (coverage) versus abundance ranking

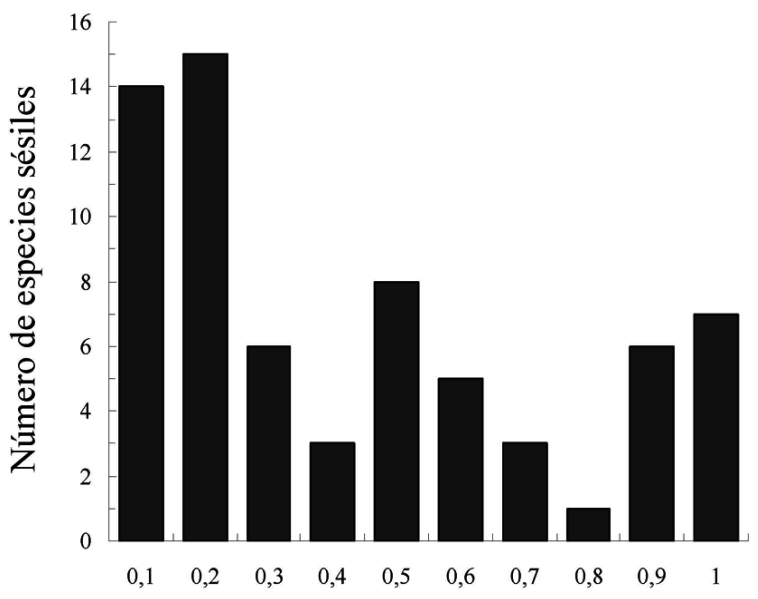

Distribución (proporción de localidades ocupadas)

Figura 6

Distribución de frecuencia de la distribución (expresada en proporción) de especies sésiles en Chile centro-norte. Las especies núcleo y satélite (Hanski 1982) corresponden a aquellas ubicadas en los intervalos de clase superior

$(0,9-1)$ e inferior $(0-0,1)$ respectivamente

Frequency distribution of the distribution (proportion of occupied localities) of sessile species in northern-central

Chile. Core and satellite species (Hanski 1982) correspond to those located at the upper (0.9-1) and lower (0-0.1) class intervals, respectively 
$51,5 \%$ del total) estuvo a lo más en $30 \%$ de las localidades, indicando que la rareza es un rasgo común en estos ensambles, y un factor clave para comprender su alta variabilidad en riqueza y composición. En contraste, 14 especies $(20,6 \%)$ fueron muy comunes y estuvieron a lo menos en el $80 \%$ de las localidades, en concordancia con los altos valores de dominancia en varios ensambles (Fig. $3)$. A escala regional, las especies que ocurrieron en el $100 \%$ de las localidades fueron sólo $6(\sim 11 \%)$ en Chile norte, y $14(\sim 28 \%)$ en Chile central, la cual sería más homogénea en su composición, como señalan otros resultados más adelante.

A escala geográfica, las especies núcleo y satélite fueron respectivamente el 20,6\% y 10,3\% del total, y sólo seis especies núcleo ocurrieron en el $100 \%$ de los ensambles: los cirripedios Jehlius cirratus (Darwin) y Notochthamalus scabrosus (Darwin), la clorófita Ulva rigida C. Agardh, las rodófitas Porphyra columbina Montagne e Hildenbrandia lecanellieri Hariot, y la anémona Phymactis clematis (Drayton). Al evaluar las especies con mayor distribución a nivel de las unidades muestrales dentro de cada ensamble, de las especies núcleo a escala geográfica sólo $U$. rigida y J. cirratus estuvieron entre las cinco primeras en $100 \%$ de los ensambles, H. lecanellieri en 77\%, y N. scabrosus en 53\%. A ellas debe agregarse el bivalvo Perumytilus purpuratus (Lamarck), que tuvo una distribución geográfica intermedia pero alcanzando muy alta ocurrencia en $47 \%$ de los ensambles. Estas últimas cinco especies son además las que alcanzaron mayor cobertura promedio a escala geográfica (ranking 1 a 5 en la Fig. 5), y en función de su ocurrencia y abundancia en todas las escalas espaciales analizadas, constituyen el grupo de especies más representativo de los ensambles sésiles de Chile centro-norte.

Por otra parte, numerosas especies mostraron una ocurrencia y cobertura consistentemente alta o baja, lo cual incidió directamente en el grado de asociación entre distribución y abundancia a distintas escalas espaciales. Esta relación fue positiva y significativa a escala geográfica $\left(\mathrm{r}_{\mathrm{S}}=0,89 ; P<0,0001 ; \mathrm{n}=68\right)$, a escala regional en Chile norte $\left(\mathrm{r}_{\mathrm{s}}=0,76 ; P<0,0001 ; \mathrm{n}=56\right) \mathrm{y}$ central $\left(\mathrm{r}_{\mathrm{S}}=0,91 ; P<0,0001 ; \mathrm{n}=51\right)$, y también a escala local en cada uno de los 17 ensambles $\left(0,80 \leq \mathrm{r}_{\mathrm{S}} \leq 0,98\right.$; $P<0,0001$ en todos los casos; $19 \leq \mathrm{n} \leq 37$ ). Los coeficientes $\mathrm{r}_{\mathrm{s}}$ fueron muy altos, $\mathrm{y}$ la forma de la relación fue en general cóncava ya que la abundancia promedio tendió a aumentar geométricamente con la distribución (como se desprende del ranking de importancia en la Fig. 5). A escala geográfica, esto se reflejó en parte en la relación negativa y significativa (Fig. 7) de la variabilidad espacial de las especies (coeficiente de variación en abundancia) con su distribución $\left(\mathrm{r}_{\mathrm{s}}=-0,92 ; P<0,0001\right.$; $\mathrm{n}=68)$, de tendencia lineal, y con su abundancia $\left(\mathrm{r}_{\mathrm{s}}=-\right.$ $0,77 ; P<0,0001 ; \mathrm{n}=68)$, con una disminución de tipo geométrico. Por ello estos patrones permiten distinguir dos grupos distintivos de especies: (a) poco abundantes, muy variables y de baja distribución; y (b) muy abundantes, poco variables y de alta distribución.
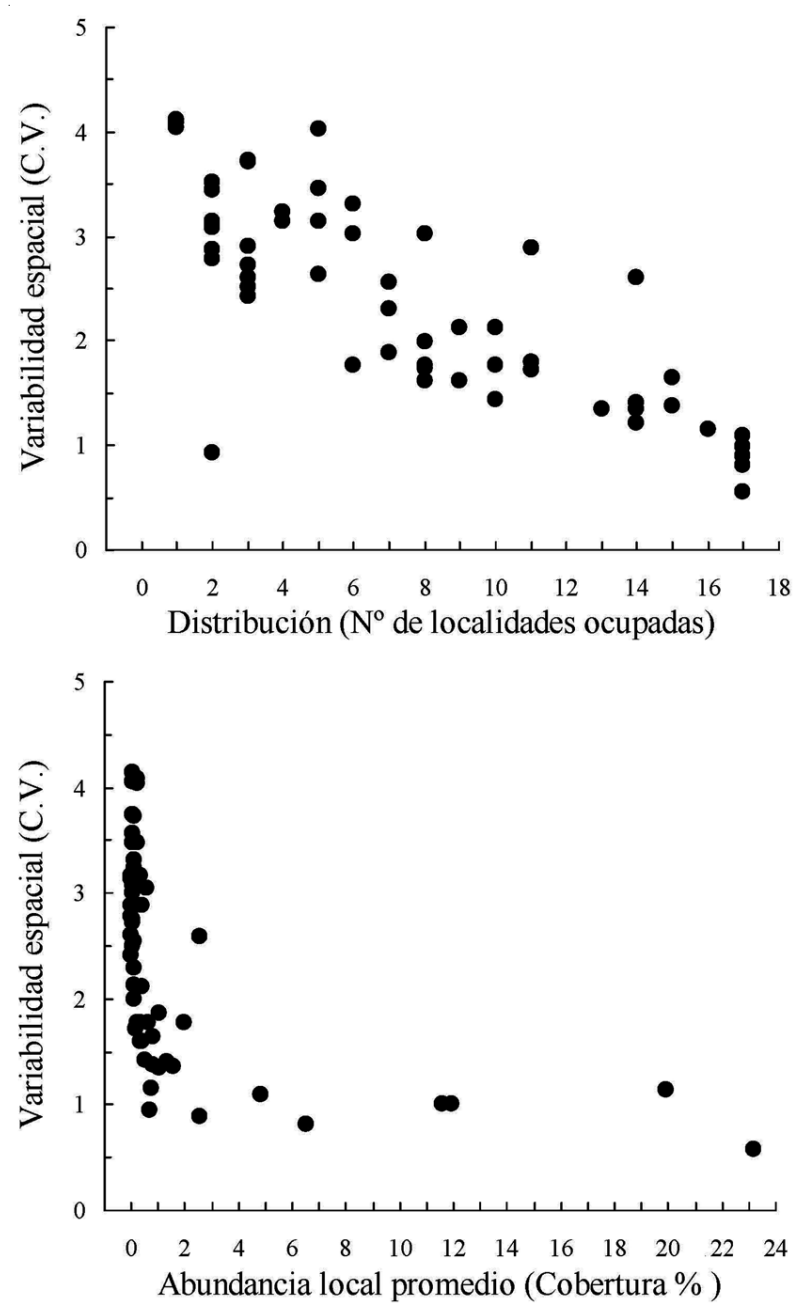

Figura 7

Relación entre la variabilidad espacial (coeficiente de variación en abundancia: C.V.) de las especies sésiles en Chile centro-norte y su distribución (figura superior) y abundancia (cobertura) local promedio (figura inferior)

Relationship between the spatial variability (coefficient of variation of abundance: C.V.) of sessile species in northerncentral Chile and their distribution (number of occupied localities; upper) and mean local abundance (coverage) (lower) 


\section{Estructura composicional}

El análisis de afinidad mostró que la similitud promedio en composición entre todos los ensambles fue de 46,1 \pm $6,7 \%$ (d.e.), o a la inversa una disimilitud de 53,9\% reflejando una alta diversidad beta en el área. En promedio ( \pm d.e.), cada ensamble incluyó $0,8 \pm 1,1$ especies exclusivas y $16,9 \pm 2,7$ especies compartidas con todos los demás. Los ensambles modales (i.e. los más representativos en diversidad) fueron Aguadita en la región norte y El Tabo en la región central, aunque ConCón y Las Cruces se ubicaron muy próximos al límite (1 d.e.) de la media de afinidad, sugiriendo que los ensambles de la región central son más redundantes en composición. En contraste, hubo cuatro sitios extremos (i.e. más diferentes), Industrias Pesqueras, Arenillas Negras, Chucumata y Yape, todos de la región norte indicando su alta heterogeneidad espacial. El valor de la diversidad de mosaico para el área fue de 3,4 indicando un nivel de complejidad del paisaje medio a bajo, aparentemente por la influencia homogeneizadora de la región central. De hecho, al analizar cada región por separado, la similitud media ( \pm d.e.) fue $44,2 \pm 7,2 \%$ en la región norte y $60,7 \pm 7,2 \%$ en la región central, con un número promedio medio ( \pm d.e.) de especies compartidas de $15,8 \pm 2,9$ y $21,1 \pm 2,1$ respectivamente. A este nivel se detectaron dos ensambles modales en la región norte (Aguadita y Los Verdes), pero ninguno en la región central indicando que sus ensambles fueron similarmente representativos (con menor diversidad beta y menor prevalencia e importancia de la rareza). Por otra parte, los ensambles extremos en el análisis por región fueron Chucumata y Arenillas Negras en Chile norte y Montemar y Los Molles en Chile central. La detección de Los Molles como anómalo es destacable ya que ha sido considerado usualmente como un sitio representativo de Chile central; sin embargo, otros análisis no incluidos en este trabajo refuerzan su importancia y su carácter singular (véase Discusión).

Por último, la Tabla 4 muestra los valores observados a escala geográfica de similitud media y diversidad de mosaico, y los generados a través de tres modelos nulos. En los tres casos la diversidad de mosaico fue significativamente distinta $(\mathrm{t} ; P<0,05)$ y menor a la esperada por rearreglos aleatorios, indicando el bajo grado de estructuración espacial de la diversidad del paisaje. En igual sentido, la similitud media fue significativamente distinta y mayor a la esperada, excepto bajo el modelo 3 $(P>0,05$; Tabla 4$)$ que simula una amplitud de distribución inherente para cada especie. Este resultado es coherente con los análisis de distribución y abundancia de especies, como se discute más adelante.
Tabla 4

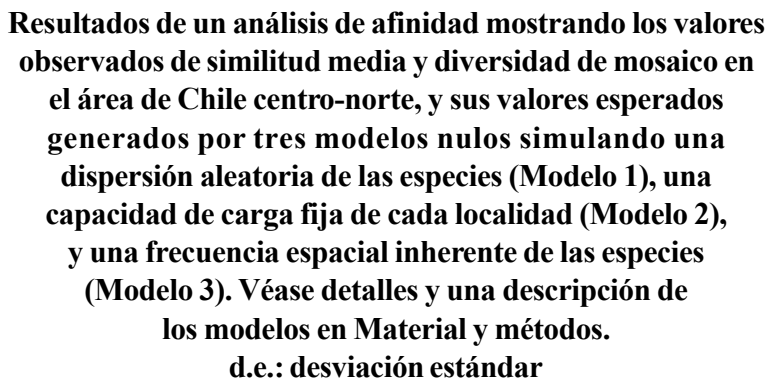

Affinity analysis results showing the observed values of mean similarity (similitud media) and mosaic diversity (diversidad de mosaico) for the central-northern Chilean area, and their expected values generated by three null models simulating a random dispersal of species (Model 1), a fixed carrying capacity of each locality (Model 2), and an inherent spatial frequency of species (Model 3). See details and description of models in Material and methods. Observado: observed; Media: mean; d.e.: standard deviation

\begin{tabular}{llccc}
\hline & Observado & Modelo 1 & Modelo 2 & Modelo 3 \\
\hline Similitud media & Media: 0,461 & 0,247 & 0,245 & 0,459 \\
& d.e.: 0,067 & 0,005 & 0,005 & 0,0009 \\
Diversidad de mosaico & Media: 3,383 & 6,809 & 6,532 & 6,622 \\
& d.e.: 0,591 & 0,611 & 0,387 & 0,631 \\
\hline
\end{tabular}

\section{Discusión}

Las especies sésiles fueron el componente mayoritario en el intermareal rocoso de Chile centro-norte, en una proporción promedio de $65 \%$ que no varió más de $10 \%$ entre ensambles y fue independiente de su ubicación geográfica. Ninguno de los atributos de diversidad medidos a nivel del ensamble completo (riqueza, uniformidad, dominancia) mostró una tendencia latitudinal aparente ni diferencias atribuibles a efectos regionales, y su variación espacial en el área de estudio fue explicada entre 83 y $100 \%$ sólo por factores locales. Esto ciertamente no significa que algún nivel espacial intermedio (e.g., mesoescala) sea irrelevante, como demuestran otros estudios (e.g., Nielsen \& Navarrete 2004). Adicionalmente, al evaluar la riqueza específica a nivel de la unidad muestral, esta escala explicó casi el doble de varianza que la escala local, y 12 veces más que la escala regional. Estimaciones similares del efecto de la escala sobre la riqueza en otros sistemas litorales no muestran un patrón común, y en muchos casos (e.g., Archambault \& Bourget 1996, Smith \& Simpson 2002, Reichert et al. 2008) las escalas pequeñas explican una fracción muy baja de la varianza, especialmente para las macroalgas, que son el componente mayoritario en los ensambles de Chile centro-norte. En este caso, parte de la relevancia de la pequeña escala se asociaría a la 
estabilización de la proporción de especies sésiles a medida que la riqueza total alcanza sus valores más altos. En este sentido, la relación entre riqueza promedio intra ensamble y riqueza total del ensamble (análogo localregional) fue positiva pero curvilineal, sugiriendo procesos de saturación a pequeña escala, similar a lo observado por Rivadeneira et al. (2002) en ensambles herbívoros intermareales entre $18^{\circ}$ y $42^{\circ} \mathrm{S}$. No obstante, algunos estudios de multiescala referidos a un verdadero pool regional detectan principalmente relaciones lineales a diferentes escalas, y otros indican que la probabilidad de registrar un patrón curvilineal donde no hay saturación real (= pseudosaturación) aumenta a escalas más pequeñas (e.g., Srivastava 1999, Witman et al. 2004, Cornell et al. 2008). De hecho la curvilinealidad a pequeña escala en este trabajo correspondería en parte a pseudosaturación por efecto de la acumulación tridimensional de organismos por unidad de área (Cornell et al. 2008), que sin embargo involucraría una limitación estructural real que puede estar sujeta a control biológico además de físico. Por ello se sugiere que la eventual existencia de una 'capacidad de carga' a nivel de parches dentro de una comunidad es un fenómeno potencialmente importante.

A nivel geográfico, la curva de dominancia-diversidad de las especies sésiles mostró una variación mayor a cuatro órdenes de magnitud en importancia, aunque sin la moda típica de una lognormal en el rango intermedio de importancia, lo cual no sería un artefacto muestral ya que el número de localidades fue suficiente para evitar una caracterización geográfica sesgada (Belasky 1992). En esta curva destacó la alta dominancia de un pequeño grupo de especies y la prevalencia de las especies raras, lo que también se reflejó en la frecuencia de distribución de las especies, con dos grupos contrastantes de muy alta y muy baja distribución, y en la relación positiva entre distribución y abundancia en todas las escalas analizadas. Un análisis paralelo de la distribución de abundancia para el mismo conjunto de especies (Camus 1998) y a las mismas escalas, registró sólo una moda desproporcionada en la clase inferior de abundancia (que incluyó casi 90\% de las especies a escala geográfica), y que sería un fenómeno real y no una 'línea de velo' muy desplazada a la derecha. Otros análisis (Camus 1998) sugieren que modelos como la distribución gama (e.g., Hastings \& Peacock 1975) serían más adecuados que el lognormal para describir la estructura de abundancia en este sistema, como se observa en otras comunidades marinas sésiles (Schmidt \& Garbutt 1985). Un análisis en especies pelágicas de giros oceánicos (copépodos y fitoplancton; McGowan \& Walker 1993) mostró esencialmente el mismo patrón de dominancia-diversidad, con una dominancia de especies raras y muy raras que conservaron este carácter en el tiempo y a distintas escalas espaciales, y tendieron a mantener el mismo ranking de abundancia. En este trabajo las especies más comunes y raras (núcleo y satélite) también tendieron a ser las mismas en distintas escalas (aunque las últimas fueron algo más variables en su ranking de abundancia; Camus 1998). Por ello algunas predicciones teóricas clásicas no aplicarían a este sistema, como la fluctuación temporal de las especies entre categorías extremas de distribución y abundancia debido a dinámicas regionales (Hanski 1982), o la alteración continua de su jerarquía de dominancia debido a las perturbaciones (Sousa 1979). En el caso pelágico, la variación del ranking de especies fue tan baja que McGowan y Walker (1993) la usaron incluso como criterio de estabilidad del sistema, pero no es claro que esto sea válido en el caso de Chile centro-norte.

Adicionalmente, estudios experimentales simultáneos (Camus \& Lagos 1996, Camus 1998) en dos ensambles del norte (Chanavayita y Yape) sugieren que la diferencia entre especies raras y comunes se debería a factores dinámicos. Las especies con mayor distribuciónabundancia local y geográfica como U. rigida, J. cirratus y $N$. scabrosus, muestran también una mayor tasa de incremento y renovación poblacional, mayor frecuencia e intensidad de reclutamiento, menor probabilidad de extinción en parches, y mayor rapidez en colonizar espacios disponibles y capturarlos a través de la sucesión. Las especies raras muestran el patrón opuesto, tendiendo a colonizar en baja frecuencia e intensidad y a comportarse como efímeras en la sucesión, con patrones de presenciaausencia muy variables. Además, la frecuencia espacial y temporal de las especies sésiles se correlacionaron positivamente dentro de cada localidad y también entre ambas localidades (Camus \& Lagos 1996, Camus 1998). Lo anterior es coherente con las estimaciones de variabilidad espacial en este trabajo, donde sólo las especies con mayor abundancia local promedio son también abundantes en todos los ensambles. En contraste, todas las especies raras son espacialmente variables en abundancia, pero siguiendo dos patrones básicos (sensu Schoener 1987): abundancia homogéneamente baja a través del espacio (rareza 'esparcida'), versus baja abundancia en varios lugares pero alta en otros (rareza difusiva). En términos de membresía comunitaria, por tanto, los ensambles sésiles de Chile centro-norte incluirían dos componentes clave por distintas razones: (a) especies persistentemente raras, que tendrían menor importancia en los procesos comunitarios pero serían determinantes en las diferencias en diversidad entre ensambles; (b) especies persistentemente comunes, que formarían el núcleo estructural de los ensambles y serían 
la base de su composición taxonómica. En este sentido, al menos parte de los patrones geográficos de diversidad observados podría ser un reflejo de procesos de pequeña escala espacial operando al interior de los ensambles, si bien estos mismos pueden a su vez estar determinados por factores más complejos y de mayor escala (e.g., véase Navarrete et al. 2008).

En relación con lo anterior, la composición específica de los ensambles sésiles fue el único aspecto analizado que reveló diferencias evidentes entre las regiones norte y central, la primera con un grado mayor de diversidad beta y rareza. El análisis de afinidad mostró que los patrones observados no fueron distintos a los predichos por el modelo nulo que simuló una distribución inherente de las especies, sugiriendo que la dinámica geográfica especie-específica podría ser un factor relevante en la composición de estos ensambles. Esto resalta la importancia de explorar la dinámica metacomunitaria (e.g., Leibold et al. 2004, Navarrete et al. 2008) en cuanto a procesos de ensamblaje e interacción biótica y su acoplamiento espacial vía dispersión. En este contexto, la dinámica sucesional local en sistemas intermareales también puede ser una determinante relevante de los procesos metacomunitarios (véase Guichard \& Steenweg 2008), como fue sugerido antes para el caso de Chile centro-norte, lo cual no elimina la importancia de los factores de mayor escala. Por ejemplo, aún debe evaluarse el posible rol del enriquecimiento regional sobre la diversidad comunitaria, cuya influencia se ha demostrado en otras áreas incluyendo la zona austral de Chile (Whitman et al. 2004). Por otra parte, en este trabajo el efecto de los factores biogeográficos sobre la composición comunitaria fue claro en casos como el ensamble de Los Molles, incluido inicialmente en Chile central, pero que resultó más afín a la región norte. Análisis paralelos (Camus 1998) confirmaron que Los Molles tiene más similitud composicional con sitios lejanos en la región norte (e.g. Mejillones) que con sitios vecinos en Chile central, y se ubicaría en una zona transicional asociada al término de la Provincia Peruana. Esto demuestra el carácter biogeográfico mixto del área de Chile centronorte y su relevancia para explicar las diferencias en composición entre sus ensambles, pero también indica que los patrones observados en otros componentes de la diversidad (riqueza, uniformidad) serían en gran medida independientes de estos efectos.

\section{Agradecimientos}

El financiamiento otorgado por CONICYT (proyecto FONDECYT No 1900770, complementado con $\mathrm{N}^{\mathrm{o}}$ 1930183) permitió generar la información presentada en este trabajo, que fue parte de la tesis doctoral del autor.
Por ello agradezco a Juan Carlos Castilla (tutor de mi tesis) por su paciencia y la herencia Lamarckiana de su respeto por la naturaleza, y también la invitación de la editora de la RBMO, que fue una oportunidad para recuperar esta investigación. Agradezco también los interesantes comentarios de un revisor anónimo, y la valiosa asistencia en terreno de Ernesto Vásquez, Enrique Vásquez, Leonora Galaz, Nelson Lagos y Marcel Duhart, y de Arturo H. Navarrete en la preparación de las figuras.

\section{Literatura citada}

Archambault P \& E Bourget. 1996. Scales of coastal heterogeneity and benthic intertidal species richness, diversity and abundance. Marine Ecology Progress Series 136: 111-121.

Belasky P. 1992. Assessment of sampling bias in biogeography by means of a probabilistic estimate of taxonomic diversity: application to modern Indo-Pacific reef corals. Palaeogeography, Palaeoclimatology, Palaeoecology 99: 243-270.

Broitman BR, SA Navarrete, F Smith \& SD Gaines. 2001. Geographic variation of southeastern Pacific intertidal communities. Marine Ecology Progress Series 224: 21-34.

Bros WE \& BC Cowell. 1987. A technique for optimizing sample size. Journal of Experimental Marine Biology and Ecology 114: 63-71.

Camus PA. 1994. Recruitment of the intertidal kelp Lessonia nigrescens Bory in northern Chile: Successional constraints and opportunities. Journal of Experimental Marine Biology and Ecology 184: 171-181.

Camus PA. 1998. Estructura espacial de la diversidad en ensambles sésiles del intermareal rocoso de Chile centronorte: la diversidad local como un resultado de determinantes de multiescala. Tesis doctoral, Departamento de Ecología, Pontificia Universidad Católica de Chile, Santiago de Chile, 276 pp.

Camus PA. 2001. Biogeografía marina de Chile continental. Revista Chilena de Historia Natural 74: 587-617.

Camus PA. 2008. Understanding biological impacts of ENSO on the eastern Pacific: An evolving scenario. International Journal of Environment and Health 2: 5-19.

Camus PA \& FP Ojeda. 1992. Scale-dependent variability of density estimates and morphometric relationships in subtidal stands of the kelp Lessonia trabeculata in northern and central Chile. Marine Ecology Progress Series 90: 193200.

Camus PA \& YN Andrade. 1999. Diversidad de comunidades intermareales rocosas del norte de Chile: El supuesto del enriquecimiento por efecto de la surgencia costera. Revista Chilena de Historia Natural 72: 389-410.

Camus PA \& N Lagos. 1996. Variación espacio-temporal del reclutamiento en ensambles intermareales sésiles del norte de Chile. Revista Chilena de Historia Natural 69: 193-204. 
Camus PA, K Daroch \& LF Opazo. 2008. Potential for omnivory and apparent intraguild predation in rocky intertidal herbivore assemblages from northern Chile. Marine Ecology Progress Series 361: 35-45.

Castilla JC. 1981. Perspectivas de investigación en estructura y dinámica de comunidades intermareales rocosas de Chile central. II. Depredadores de alto nivel trófico. Medio Ambiente 5: 190-215.

Castilla JC. 1988. Earthquake-caused coastal uplift and its effects on rocky intertidal kelp communities. Science 242: 440-443.

Castilla JC \& PA Camus. 1992. The Humboldt-El Niño scenario: coastal benthic resources and anthropogenic influences, with particular reference to the 1982/83 ENSO. South African Journal of Marine Science 12: 111-119.

Cornell HV. 1993. Unsaturated patterns in species assemblages: the role of regional processes in setting local species richness. En: Ricklefs RE \& D Schlutter (eds), Species diversity in ecological communities. Historical and biogeographical perspectives, pp. 243-252. The University of Chicago Press, Chicago \& Londres.

Cornell HV, RH Carlson \& TP Hughes. 2008. Local-regional species richness relationships are linear at very small to large scales in west-central Pacific corals. Coral Reefs 27: 145-151.

Dethier MN, ES Graham, S Cohen \& LM Tear. 1993. Visual versus random-point percent cover estimations: 'objective' is not always better. Marine Ecology Progress Series 96: 93-100.

Fernández M, E Jaramillo, PA Marquet, CA Moreno, SA Navarrete, FP Ojeda, CR Valdovinos \& JA Vásquez. 2000. Diversity, dynamics and biogeography of Chilean benthic nearshore ecosystems: an overview and guidelines for conservation. Revista Chilena de Historia Natural 73: 797-830.

Guichard F \& R Steenweg. 2008. Intrinsic and extrinsic causes of spatial variability across scales in a metacommunity. Journal of Theoretical Biology 250: 113-124.

Hanski I. 1982. Dynamics of regional distribution: the core and satellite species hypothesis. Oikos 38: 210-221.

Hastings NAJ \& JB Peacock. 1975. Statistical distributions. 130 pp. Halsted Press, Nueva York.

Hill MO. 1973. Diversity and evenness: a unifying notation and its consequences. Ecology 54: 427-431.

Hurlbert SH. 1984. Pseudoreplication and the design of ecological field experiments. Ecological Monographs 54: 187-211.

Ibáñez CM, PA Camus \& FJ Rocha. 2008. Diversity and distribution of cephalopod species off the coast of Chile. Marine Biology Research: en prensa.

Istock CA \& SM Scheiner. 1987. Affinities and high-order diversity within landscape mosaics. Evolutionary Ecology 1: 11-29.

Jaksic FM. 1981. Use and abuse of the term 'guild' in ecological studies. Oikos 37: 397-400.
Lagos NA, JC Castilla \& BR Broitman. 2008. Spatial environmental correlates of intertidal recruitment: a test using barnacles in northern Chile. Ecological Monographs 78: 245-261.

Lagos NA, SA Navarrete, F Véliz, A Masuero \& JC Castilla. 2005. Meso-scale spatial variation in settlement and recruitment of intertidal barnacles along the coast of central Chile. Marine Ecology Progress Series 290: 165-178.

Leibold MA, M Holyoak, N Mouquet, P Amarasekare, JM Chase, MF Hoopes, RD Holt, JB Shurin, R Law, D Tilman, R Loreau \& A Gonzalez. 2004. The metacommunity concept: a framework for multi-scale community ecology. Ecology Letters 7: 601-613.

Littler MM \& DS Littler. 1980. The evolution of thallus form and survival strategies in benthic marine macroalgae: field and laboratory tests of a functional form model. American Naturalist 116: 25-44.

Lohse DP. 1993. The importance of secondary substratum in a rocky intertidal community. Journal of Experimental Marine Biology and Ecology 166: 1-17.

Ludwig JA \& JF Reynolds. 1988. Statistical ecology. A primer on methods and computing, 337 pp. John Wiley \& Sons, Nueva York.

Magurran AE. 1988. Ecological diversity and its measurement, 413 pp. Princeton University Press, Princeton.

McGowan JA \& PW Walker. 1993. Pelagic diversity patterns. En: Ricklefs RE \& D Schluter (eds), Species diversity in ecological communities. Historical and geographical perspectives, pp. 203-214. The University of Chicago Press, Chicago, Illinois.

Menge BA \& AM Olson. 1990. Role of scale and environmental factors in regulation of community structure. Trends in Ecology and Evolution 5: 52-57.

Moreno CA, G Asencio, WE Duarte \& V Marín. 1998. Settlement of the Muricid Concholepas concholepas, and its relationship with El Niño and coastal upwelling in southern Chile. Marine Ecology Progress Series 167: 171175 .

Navarrete SA, BR Boitman \& BA Menge. 2008. Interhemispheric comparison of recruitment to intertidal communities: pattern persistence and scales of variation. Ecology 89: 1308-1322.

Navarrete SA, BR Broitman, EA Wieters, GR Finke, RM Venegas \& A Sotomayor. 2002. Recruitment of intertidal invertebrates in the southeast Pacific: Interannual variability and the 1997-1998 El Niño. Limnology and Oceanography 47: 791-802.

Nielsen KJ \& SA Navarrete. 2004. Mesoscale regulation comes from the bottom- up: Intertidal interactions between consumers and upwelling. Ecology Letters 7: 31- 41.

Pielou EC. 1976. Population and community ecology: principles and methods, $424 \mathrm{pp}$. Gordon and Breach Science Publishers, Nueva York.

Preston FW. 1948. The commonness, and rarity, of species. Ecology 29: 254-283. 
Reichert K, F Buchholz, I Bartsch, T Kersten \& L Giménez. 2008. Scale-dependent patterns of variability in species assemblages of the rocky intertidal at Helgoland (German Bight, North Sea). Journal of the Marine Biological Association UK 88: 1319-1329.

Rickelfs RE \& D Schluter. 1993. Species diversity in ecological communities. Historical and geographical perspectives, $414 \mathrm{pp}$. The University of Chicago Press, Chicago \& Londres.

Rivadeneira MM, M Fernández \& SA Navarrete. 2002. Latitudinal trends of species diversity in rocky intertidal herbivore assemblages: spatial scale and the relationship between local and regional species richenss. Marine Ecology Progress Series 245: 123-131.

Santelices B. 1980. Phytogeographic characterization of the temperate coast of Pacific South America. Phycologia 19: $1-12$.

Santelices B, S Montalva \& P Oliver. 1981. Competitive algal community organization in exposed habitats from central Chile. Marine Ecology Progress Series 6: 267-276.

Santelices B \& FP Ojeda. 1984. Recruitment, growth and survival of Lessonia nigrescens Bory (Phaeophyta) at various tidal levels in exposed habitats of central Chile. Marine Ecology Progress Series 19: 73-82.

Scheiner SM. 1992. Measuring pattern diversity. Ecology 73: 1860-1867.

Schmidt GH \& DJ Garbutt. 1985. Species abundance data from fouling communities conform to the gamma distribution. Marine Ecology Progress Series 23: 287-290.

Schoener TW. 1987. The geographical distribution of rarity. Oecologia 74: 161-173.

Smith SDA \& RD Simpson. 2002. Spatial variation in the community structure of intertidal habitats at Macquarie Island (sub-Antarctic). Antarctic Science 14: 374-384.

Sokal RR \& FJ Rohlf. 1995. Biometry: The principles and practice of statistics in biological research, $887 \mathrm{pp}$. WH Freeman and Company, Nueva York.

Srivastava DS. 1999. Using local-regional richness plots to test for species saturation: pitfalls and potentials. Journal of Animal Ecology 68: 1-16.
Sousa WP. 1979. Experimental investigations of disturbance and succession in a rocky intertidal algal community. Ecological Monographs 49: 227-254.

Takesue RK, A van Geen, JD Carriquiry, E Ortiz, L Godinez-Orta, I Granados, M Saldívar, L Ortlieb, R Escribano, N Guzmán, JC Castilla, M Varas, M Salamanca \& C Figueroa. 2004. Influence of coastal upwelling and El Niño-Southern Oscillation on nearshore water along Baja California and Chile: Shore-based monitoring during 1997-2000. Journal of Geophysical Research 109: C03009.1-C03009.14.

Thiel M, E Macaya, E Acuña, W Arntz, H Bastias, K Brokordt, PA Camus, JC Castilla, LR Castro, M Cortés, CP Dumont, R Escribano, M Fernandez, DA Lancellotti, JA Gajardo, CF Gaymer, I Gomez, AE González, HE Gonzalez, PA Haye, JE Illanes, JL Iriarte, G LunaJorquera, C Luxoro, PH Manriquez, V Marín, P Muñoz, SA Navarrete, E Perez, E Poulin, J Sellanes, A Sepúlveda, W Stotz, F Tala, A Thomas, CA Vargas, JA Vasquez, A Vega. 2007. The Humboldt Current System of northerncentral Chile: oceanographic processes, ecological interactions and socio-economic feedback. Oceanography and Marine Biology: An Annual Review 45: 195-345.

Vásquez JA, PA Camus \& FP Ojeda. 1998. Diversidad, estructura y funcionamiento de ecosistemas litorales rocosos del norte de Chile. Revista Chilena de Historia Natural 71: 479-499.

Wieters EA. 2005. Upwelling control of positive interactions over mesoscales: A new link between bottom-up and topdown processes on rocky shores. Marine Ecology Progress Series 301: 43-54.

Witman JD, RJ Etter \& F Smith. 2004. The relationship between regional and local species diversity in marine benthic communities: a global perspective. Proceedings of the National Academy of Sciences (USA) 101: 1566415669.

Wolda H \& J Marek. 1994. Measuring variation in abundance, the problem with zeros. European Journal of Entomology 91: 145-161. 